\title{
Qualidade na Elicitação de Requisitos: um estudo de caso sobre a participação da equipe
}

\author{
Requirements Elicitation Quality: a case study on team participation \\ Naiara Crislaine Alflen*1, Edmir Parada Vasques Prado ${ }^{1}$ \\ ${ }^{1}$ Universidade de São Paulo, São Paulo, Brasil.
}

\begin{tabular}{l}
\hline I N F O A R T I G O \\
\hline Palavras-chave: \\
Elicitação de Requisitos, \\
Sistemas de Informação, \\
Participação da Equipe.
\end{tabular}

ARTICLE INFO

\section{Keywords:}

Requirements Elicitation.

Information Systems.

Team Participation.

\begin{abstract}
R E S U M O
A elicitação de requisitos (ER) é uma tarefa difícil na qual é necessário lidar com ambiguidade de informações e dados inconsistentes. Ela busca descobrir e entender as necessidades dos usuários. O sucesso do desenvolvimento de software depende da elicitação correta dos requisitos, e a qualidade deles é influenciada pela participação da equipe no processo de elicitação. O objetivo desta pesquisa é analisar a participação da equipe na qualidade da elicitação de requisitos funcionais (RF) e requisitos não funcionais (RNF). Foi realizado um estudo de caso em um curso de Sistemas de Informação, envolvendo 53 alunos. Os resultados confirmaram que a participação de todos os membros da equipe no processo de ER melhora a completude e a consistência dos RF e RNF. Além disso, foi identificada uma associação positiva entre motivação e comunicação da equipe com os resultados da pesquisa.
\end{abstract}

A B S T R A C T
Requirements elicitation (RE) is a difficult task in which there are issues
related to information ambiguity, incomplete and inconsistent data. It seeks to
discover and understand the users' needs. The success of software
development depends on the correct elicitation of requirements, and their
quality is influenced by the team's participation in the elicitation process. This
research aims to analyze the contribution of team's participation in the
elicitation of functional requirements (FR) and non-functional requirements
(NFR). A case study was carried out in an Information Systems course,
involving 53 students. The outcome confirmed that the participation of all
team members in the RE process improves the completeness and consistency
of RF and RNF. In addition, a positive association was identified between
team motivation and communication with the research results.

\section{Introdução}

A engenharia de requisitos é a fase mais complexa no desenvolvimento de software (ROMERO; VIZCAÍNO; PIATTINI, 2009; PANDEY et al., 2010; FERNANDES et al., 2012; BUITRON, et al., 2018; MARIA; ALI, 2018). Uma das etapas da engenharia de requisitos é a elicitação de requisitos (ER), que é dedicada a identificação do problema e necessidade dos usuários (PRESSMAN, 2016).

Segundo Hickey e Davis, 2003 e Vijayan et al., 2016 a elicitação de requisitos é o entendimento da real necessidade do usuário, uma tarefa difícil na qual é necessário lidar com ambiguidade de informações, dados incompletos e inconsistentes, em que os requisitos não são claramente conhecidos. Nesse contexto, a elicitação de

\footnotetext{
* Correspondência para autor:

naiara.alflen@gmail.com (Alflen, N.C.) (ORCID: 0000-0001-9666-1417), eprado@usp.br (Prado, E.P.V.) (ORCID: 00000002-3505-6122).
}

1679-1827 (C) 2019 Gest@o.org. 
requisitos não se refere apenas a escrever requisitos, mas em descobrir e entender o real problema e as necessidades dos usuários (ARAÚJO; ANJOS; SILVA, 2015). Como apresentado por Gonzales e Leroy, 2011, a incompreensão da necessidade do usuário é um dos principais fatores do fracasso de um projeto e ainda, Nuseibeh e Easterbrook, 2000 argumentam que na maioria das vezes os usuários têm dificuldade de expressar seus requisitos. De forma semelhante, Mishra et al. (2008) apresentam que a maior falha em projetos de software são requisitos incompletos e incorretos.

A literatura destaca vários fatores de sucesso em projetos de desenvolvimento de software: Babar, Bunker e Gill (2018) citam que o sucesso do software depende da elicitação correta dos requisitos; e Hidalga et al. (2016) destacam o engajamento do usuário e a identificação precoce de problemas como fatores de sucesso. De forma semelhante, diversos autores têm mostrado a importância da participação do usuário na ER (CASTRO-HERRERA et al., 2009; SEYFF et al., 2015; KATINA et al., 2014; ANGELIS et al., 2018).

A motivação desta pesquisa se relacionada à participação da equipe do projeto como fator de sucesso na ER. Esta investigação encontra respaldo no trabalho de Knauss et al. (2018) que destacam a importância da participação da equipe na ER em projetos de desenvolvimento de software. Dentro desse contexto, o objetivo desta pesquisa é analisar a participação da equipe na qualidade de elicitação de requisitos funcionais (RF) e requisitos não funcionais (RNF).

\section{Referencial Teórico}

O referencial teórico desta pesquisa inicia abordando os conceitos básicos sobre ER, em especial os requisitos funcionais e não funcionais. Em seguida, são apresentados os tópicos relacionados à participação da equipe no processo de ER.

\subsection{Elicitação de Requisitos}

A elicitação de requisitos (ER) é uma das etapas da engenharia de requisitos (SOMMERVILLE, 2016; LAMSWEERDE, 2000; HOFMANN; LEHNER, 2001; NUSEIBEH; EASTERBROOK, 2000). Para Pacheco e Garcia (2012), a elicitação de requisitos é uma das etapas mais críticas do desenvolvimento de software e que segundo Curcio et al., 2018 e Batool et al., 2013 é a descoberta do sistema e suas limitações, por meio da comunicação com os stakeholders.

Os stakeholders são as partes envolvidas no processo da ER, com diferentes conhecimentos, necessidades, preocupações, habilidades e meios de expressão (LAMSWEERDE, 2000). Podem fazer parte do grupo de stakeholders os clientes, usuários, gerentes de projeto, analistas, desenvolvedores (BOURQUE; FAIRLEY, 2014; HOFMANN; LEHNER, 2001; LAMSWEERDE, 2000). Segundo Pacheco e Garcia (2012), a identificação correta dos stakeholders melhora as atividades de ER. Isto porque, o principal objetivo da ER é descobrir qual problema precisa ser resolvido (NUSEIBEH; EASTERBROOK, 2000) e compreender as necessidades de diferentes stakeholders (BOURQUE; FAIRLEY, 2014). Assim, é importante entender em qual contexto o software será usado para facilitar sua implementação (BATOOL et al., 2013).

Por outro lado, Mishra et al. (2008) argumentam que a maior falha em projetos de software são requisitos incompletos e incorretos. De forma similar, Sadiq et al. (2009) e Gonzales e Leroy (2011) apontam a especificação inadequada dos requisitos como a responsável por $70 \%$ dos erros de um software. Um dos fatores que influenciam a qualidade dos requisitos são as técnicas de ER (HICKEY; DAVIS, 2003), pois é nesta fase que ocorre o aprendizado e a comunicação com os usuários.

Segundo Pandey et al. (2010), existem dois tipos de requisitos: funcionais e não funcionais.

\subsection{Requisitos Funcionais}

No contexto de desenvolvimento de software, segundo Sommerville, 2016 e Alvertis et al., 2016 os requisitos funcionais (RF) descrevem o que o software deve fazer, ou o que se espera que o software faça. Nesse caso, os RF 
expressam as necessidades dos usuários e são as atividades que o software deve executar e como apontado por Buitron et al., 2018 sem a preocupação das limitações físicas. Esses requisitos descrevem em detalhes as funções do software, com suas entradas, saídas e exceções (SOMMERVILLE, 2016).

A descrição de um RF deve ser clara e precisa, e realizada de forma abstrata para entendimento do usuário. Além disso, os requisitos devem ser de fácil entendimento para que desenvolvedores possam implementá-los corretamente. A especificação dos requisitos deve ser completa e consistente (SOMMERVILLE, 2016). Completude é a definição e descrição de todos os serviços solicitados (BOEHM, 2000), e consistência significa que as definições dos requisitos não podem deixar dúvidas e nem ter contradição entre eles.

\subsection{Requisitos Não Funcionais}

Na elicitação de RNF são listados os requisitos de como o software executará as atividades (ALVERTIS et al., 2016). É um estágio importante para a seleção de tecnologia, alocação de hardware e definição de padrões de desenvolvimento (YOUNAS et al., 2017). De acordo com Pandey et al., 2010, os RNF especificam as propriedades do software e ainda, segundo Buitron et al., 2018, os RNF limitam o comportamento do software especificando seus atributos. Além disso, na elicitação dos RNF são definidos os mecanismos de segurança do software, distribuição e licenças (YOUNAS et al., 2017).

Os RNF descrevem a qualidade do software (BOURQUE; FAIRLEY, 2014) e podem levar a falha um projeto, caso não sejam bem especificados (ASGHAR et al., 2017). Por isso, os critérios de qualidade podem ser considerados no processo de elicitação de requisitos não funcionais (YOUNAS et al., 2017).

\subsection{Participação da Equipe}

Pacheco e Garcia (2012) argumentam que a principal atividade, antes de iniciar a elicitação de requisitos, é a correta identificação dos envolvidos, pois auxilia numa participação mais efetiva. Equipe de desenvolvimento e cliente no mesmo ambiente, seja físico ou virtual, facilita para que todos os envolvidos tenham a mesma visão do que será desenvolvido. Franken et al., (2015) afirmam que uma participação próxima entre a equipe de desenvolvimento e o cliente, durante todo o ciclo de vida de um projeto, torna-se um fator de sucesso. Em complemento a isso, Melo et al., (2011) afirmam que para a participação mais efetiva deve-se trabalhar com equipes menores, prática já conhecida em ambientes ágeis. Entretanto, a participação do cliente nos projetos de software com metodologias ágeis, muitas vezes, não é realizada com a participação de toda a equipe que desenvolverá o software. Franken et al., (2015) mencionam que a participação do cliente nos projetos tende a ser apenas com o Product Owner (PO), que se torna o meio de comunicação entre a equipe de desenvolvimento e o cliente.

Motivação e comunicação também são fatores importantes no processo de desenvolvimento de software. A motivação, segundo Melo et al. (2011), é um fator crítico para o sucesso de um projeto, desenvolvedores desmotivados tendem a não estarem presentes nas atividades de um projeto. Além disso, Väätäjä et al., (2016) afirmam que a motivação da equipe reflete nos benefícios ao cliente.

Segundo Batool et al., (2013) um dos benefícios das metodologias ágeis é a constante comunicação entre os membros da equipe do projeto, e entre a equipe e o cliente. Reforçando essa ideia, Curcio et al. (2018) afirmam que uma equipe ágil utiliza da colaboração e da comunicação para executar as atividades da ER. Apesar das metodologias ágeis incentivarem a comunicação direta entre a equipe, as características individuais de cada membro da equipe influenciam na comunicação. Liu et al., (2011) mencionam que conflitos interpessoais impactam na elicitação e priorização dos requisitos e sob o mesmo ponto de vista Marshall e Gamble (2015) afirmam que a comunicação e os conflitos influenciam direta ou indiretamente no resultado de um projeto, que podem ser positivos ou negativos.

Outro ponto abordado nas metodologias ágeis é a comunicação entre a equipe e o cliente. Apesar de Franken et al., (2015) mencionarem que a comunicação é com o PO. El-Najar, Ahmad e Alkandari (2019) afirmam que a proposta das metodologias ágeis é a proximidade da equipe com o cliente. O principal benefício da equipe e cliente trabalharem próximos é o real entendimento das necessidades do cliente e consequentemente num produto de maior qualidade. De acordo com Younas et al. (2017) o cliente elicita seus requisitos para a equipe desenvolver. 
Posteriormente, por meio de entregas rápidas a equipe valida com o cliente a parte desenvolvida para seguir com as demais. No entanto, na comunicação da equipe com o cliente podem surgir conflitos que interferem na comunicação e por consequência no insucesso de um projeto (JIA; CAPRETZ, 2017).

\subsection{Modelo de Referência da Pesquisa}

Nesta seção é apresentada a esquematização do modelo de referência da pesquisa, as proposições de pesquisa e a descrição das variáveis que compõem as proposições. As proposições consideram apenas as três técnicas de ER mais citadas na literatura e mais usadas no contexto empresarial: entrevista, prototipagem e brainstorming.

\subsubsection{Exemplo}

O modelo de referência da pesquisa está esquematizado na Figura 1. O modelo é composto por duas proposições de pesquisa, que sugerem que a participação de todos os membros da equipe de projeto no processo de ER melhora a qualidade dos RF e RNF.

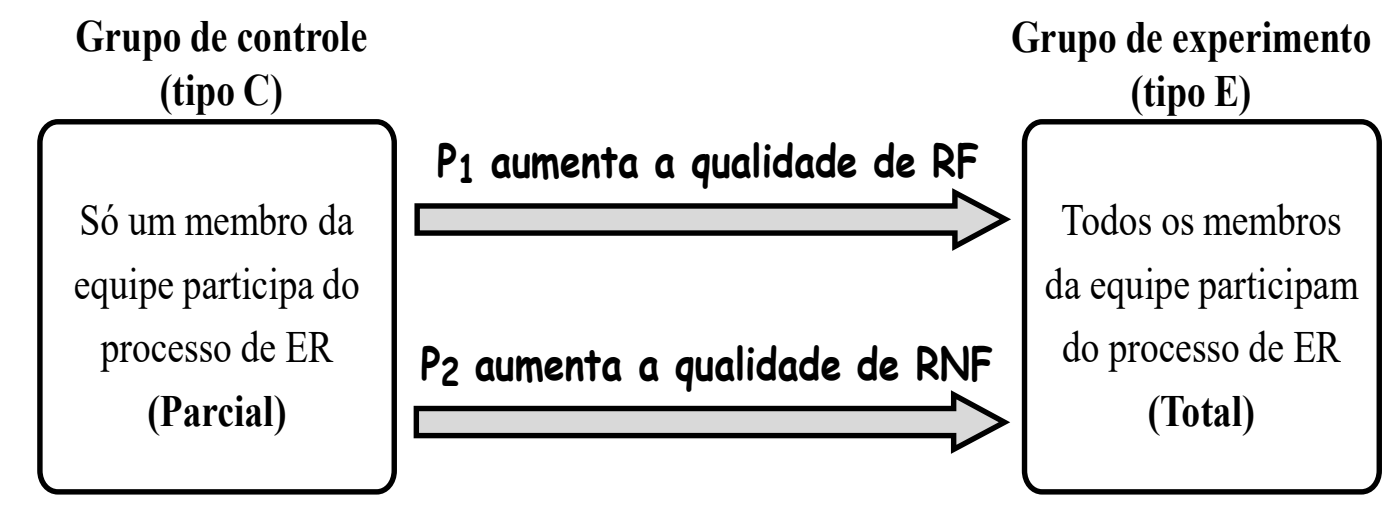

* P1 a P2 são proposições de pesquisa

Figura 1 - Modelo de referência da pesquisa.

Fonte: elaborado pelo autor.

Tipo C. As equipes que pertencem a este grupo têm uma participação parcial no processo de ER, ou seja, somente um membro da equipe (PO - Product Owner) participa das entrevistas com o cliente. Dentro da metodologia ágil, o PO é o responsável por descrever e priorizar os requisitos para a equipe de desenvolvimento, além de garantir que eles sejam completos e consistentes (BATOOL et al., 2013).

Tipo E. Nas equipes que pertencem a este grupo todos os seus membros participação do processo de ER.

\subsubsection{Proposições da Pesquisa}

O modelo de referência apresenta duas proposições a serem verificadas nesta pesquisa:

P1. Equipes com a participação de todos os membros no processo de ER produzem uma especificação de requisitos funcionais $(\mathrm{RF})$ de melhor qualidade.

P2. Equipes com a participação de todos os membros no processo de ER produzem uma especificação de requisitos não funcionais (RNF) de melhor qualidade.

Essas proposições relacionam variáveis que estão descritas a seguir. 


\subsubsection{Variáveis da Pesquisa}

O modelo de referência desta pesquisa conta com três tipos de variáreis: independentes, dependentes e de controle. Nas variáveis independentes é possível a manipulação dos dados e elas influenciam ou afetam as variáveis dependentes. Por último, as variáveis de controle são consideradas variáveis que influenciam os resultados das variáveis dependentes e pode ser utilizada para explicar os resultados (CRESWELL, 2007).

Variável independente. Representa a variável cuja manipulação permite o estudo do impacto na qualidade dos requisitos elicitados.

(1) Participação da equipe. Refere-se ao tipo de participação das equipes no processo de ER: nível 0 refere-se à participação parcial da equipe, ou seja, apenas um membro da equipe interage com o cliente (PO em projetos ágeis), e nível 1 refere-se à participação de toda a equipe nas interações com o cliente.

Variáveis dependentes. São duas variáveis que representam o nível de qualidade na elicitação dos RF e RNF. Nesta pesquisa a qualidade refere-se a dois atributos: completude e consistência dos requisitos.

(1) Qualidade dos RF. Representa a correta elicitação, em termos de completude e consistência dos RF. Esta variável apresenta três níveis:

- Nível 0. Este nível refere-se a uma ER de baixa qualidade. Apenas um terço ou menos dos RF foram elicitados com completude e consistência.

- Nível 1. Este nível refere-se a uma ER de qualidade média. Até dois terços dos RF foram elicitados com completude e consistência.

- Nível 2. Este nível refere-se a uma ER de alta qualidade. Mais de dois terços dos RF foram elicitados com completude e consistência.

(2) Qualidade dos RNF. Representa a qualidade dos RNF em termos de completude e consistência dos RF, e apresenta os mesmos níveis da variável anterior.

Variáveis de controle. O modelo de pesquisa é constituído por três variáveis de controle que estão associadas ao envolvimento das equipes no processo de ER.

(1) Motivação. Refere-se à motivação da equipe em todo o processo de ER avaliado por dois critérios: assiduidade e pontualidade da equipe nas interações. Este indicador apresenta três níveis: nível 0, que representa equipe com falta de assiduidade e falta de pontualidade; nível 1, que representa equipe com falta de assiduidade ou falta de pontualidade; e nível 2, que representa equipe com assiduidade e pontualidade. Foram utilizados os critérios assiduidade e pontualidade para medição do indicador motivação, pois segundo Grotta (2019), eles podem ser usados para avaliação da motivação de uma equipe.

(2) Comunicação. Refere-se à comunicação no processo de ER entre os membros da equipe e entre a equipe e o cliente. Este indicador apresenta três níveis: nível 0, que representa falta de comunicação entre os membros da equipe e entre a equipe e o cliente; nível 1 , que representa falta de comunicação entre os membros da equipe ou entre a equipe e o cliente; e nível 2, que representa comunicação adequada entre os membros da equipe e entre a equipe e o cliente. $\mathrm{O}$ instrumento para coleta de dados é apresentado no apêndice $\mathrm{A}$.

(3) Desempenho. Refere-se ao histórico de desempenho dos membros da equipe. No caso de equipe composta por alunos de graduação, trata-se do histórico escolar. Isto porque, Biggs e Tang (2011) sugerem a observação do histórico de desempenho dos discentes na avaliação educacional. Este indicador é constituído por uma escala racional que varia de 0 a 10 .

\section{Método de Pesquisa}

A pesquisa proposta neste trabalho caracteriza-se por ser um estudo exploratório. Segundo Cervo et al. (2007), a pesquisa exploratória visa oferecer informações sobre o objeto de estudo e orientar a formulação de hipóteses para futuras pesquisas. Esta pesquisa explora a influência da participação da equipe na qualidade dos RF e RNF. Cabe destacar ainda, que este estudo é uma pesquisa qualitativa e transversal do tipo único, pois a coleta de dados foi feita uma única vez. 

de dados.

Nesta seção são apresentadas as etapas da pesquisa e os procedimentos metodológicos para coleta e análise

\subsection{Etapas da Pesquisa}

As etapas da pesquisa estão esquematizadas na Figura 2. A primeira etapa compreende a revisão da literatura, na qual foram apresentados e descritos os conceitos básicos da pesquisa. A partir da revisão da literatura foi realizada a segunda etapa com a elaboração do modelo de referência da pesquisa composto pelas proposições e variáveis da pesquisa. Em seguida, na terceira etapa foram definidos os procedimentos metodológicos, que estão apresentados nesta seção. Na quarta etapa foi realizado o trabalho de coleta de dados que estão apresentado na seção 4 e 5 . $\mathrm{Na}$ quinta e última etapa foram realizadas as análises e as considerações finais da pesquisa.

Foi adotado o estudo de caso como estratégia de pesquisa, pois segundo Eisenhardt (1989) o estudo de caso permite ao pesquisador responder de forma flexível a novas descobertas quando da coleta de dados primários. Recomenda-se a identificação dos sujeitos da pesquisa. Entretanto, são aceitas situações em que esta identificação não é possível. Nesta pesquisa, por razões de sigilo, não serão identificados a organização e os participantes. Destaca-se, porém, que nenhuma informação relevante ao estudo foi omitida.

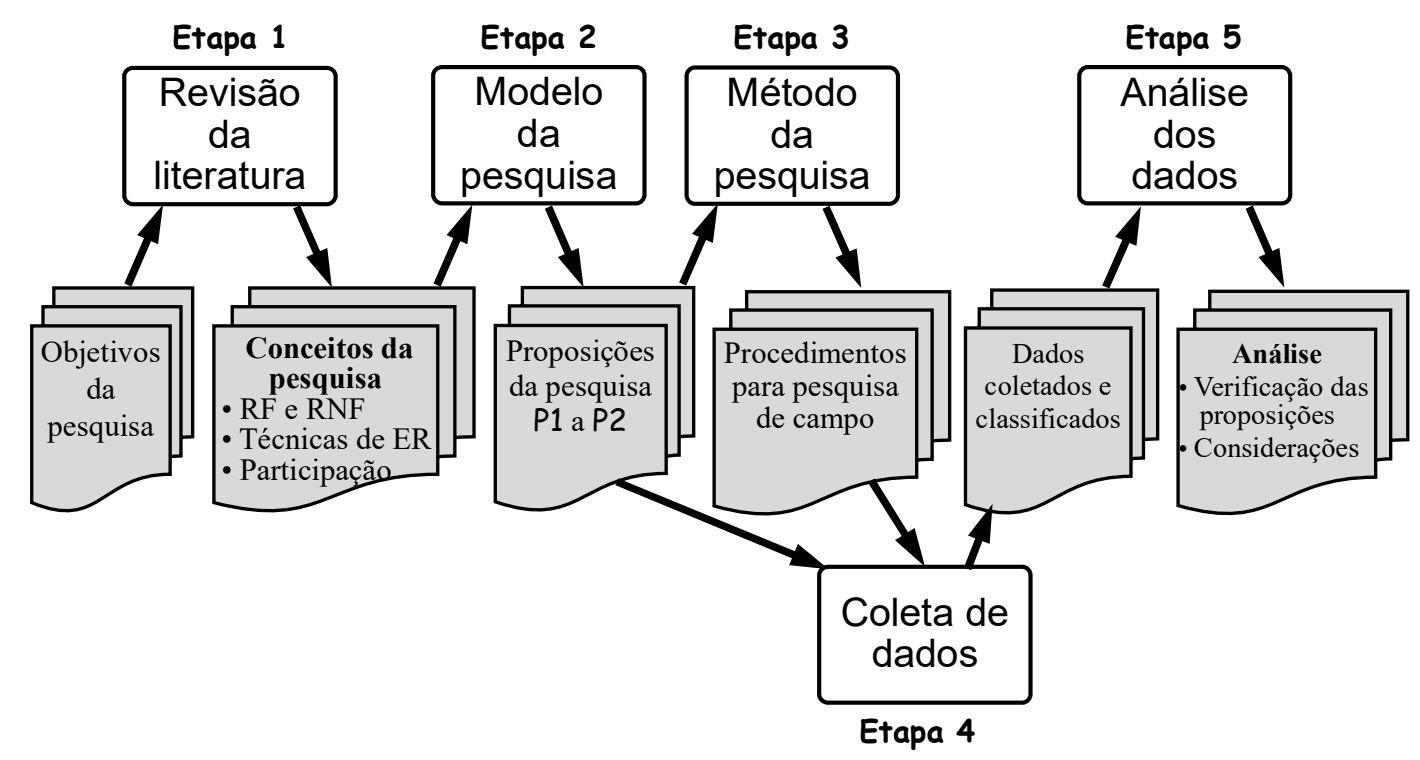

Figura 2 - Etapas da pesquisa.

Fonte: elaborado pelo autor.

\subsection{Protocolo do Estudo de Caso}

O protocolo da pesquisa descreve a seleção dos sujeitos da pesquisa. Em seguida é apresentado o sistema e seus requisitos que devem ser elicitados. Por último, é apresentado o plano de atividades e de interação com as equipes para coleta de dados.

\subsubsection{Seleção dos Sujeitos da Pesquisa}

O estudo de caso foi conduzido no segundo semestre de 2019, com alunos de um curso de graduação em Sistemas de Informação na cidade de São Paulo. Foi selecionada uma turma do terceiro semestre do curso, com aulas no 
período matutino. A escolha dessa turma possibilitou ter alunos com maior disponibilidade para as atividades discentes, pois poucos alunos faziam estágios ou outras atividades profissionais.

A turma selecionada possuía 53 alunos, número suficiente para a composição de várias equipes para o projeto de desenvolvimento de software necessárias para o estudo de caso proposto.

Escolheu-se uma disciplina obrigatória do curso e que possuía um trabalho semestral relevante dentro da carga total de horas de aula. Isso permitiu uma maior dedicação dos alunos e um melhor acompanhamento das atividades.

\subsubsection{Requisitos a Serem Elicitados}

$\mathrm{O}$ protocolo propõe às equipes a ER de um sistema de avaliação de funcionários. As equipes devem elicitar os requisitos do sistema e apresentar os resultados por meio de um protótipo funcional. Os requisitos a serem elicitados, estão resumidos na figura 3 e descritos abaixo:

(1) Requisitos funcionais: foram definidos $14 \mathrm{RF}$, que foram categorizados em quatro grupos:

- Desempenho. Esta categoria compreende: (i) eficiência, corresponde a avaliação do funcionário em relação ao resultado do seu trabalho em termos de cumprimento de prazos e orçamentos; e (ii) eficácia, representa a avaliação do funcionário em relação às suas entregas com qualidade e ao cumprimento das metas definidas pelos gestores.

- Comportamento. Esta categoria compreende: (iii) postura, que se refere à assiduidade, pontualidade $\mathrm{e}$ linguagem usada no ambiente de trabalho; (iv) comportamento sob pressão, que corresponde à avaliação das reações do funcionário a situações de carga de trabalho excessiva ou necessidade de atingir metas desafiadoras; (v) comportamento ético, que refere-se ao comportamento do funcionário ao assumir erros cometidos e ao relacionar-se com os colegas de trabalho; e (vi) comportamento em relação aos desafios, que refere-se ao comportamento do funcionário quando existe uma atividade fora do seu cotidiano que precisa ser executada, ou então, o aprendizado de um determinado tema para execução de uma nova atividade.

- Habilidades sociais e pessoais. Esta categoria compreende: (vii) resolução de problemas, refere-se à eficácia com que o funcionário resolve problemas relacionados ao seu trabalho; (viii) capacidade de comunicação, refere-se à comunicação do funcionário com os colegas de trabalho; (ix) proatividade, corresponde à avaliação do funcionário em relação à iniciativa para realização das atividades; (x) colaboração com a equipe, que compreende a disposição de compartilhar conhecimentos e realização de atividades com a equipe de trabalho; (xi) relacionamento com a equipe, refere-se a interação com a equipe na manutenção de um ambiente de trabalho adequado e em relações de empatia; (xii) aderência aos valores e diretrizes da empresa, que refere-se ao quanto o funcionário se enquadrou à cultura organizacional, à missão e visão da empresa; e (xiii) relacionamento interpessoal, refere-se à habilidade de se relacionar e interagir com os demais;

- (xiv) Relatório, refere-se ao documento gerado com os resultados das avaliações individuais do funcionário. No relatório, os resultados das avaliações de todos os requisitos anteriores deveriam ser apresentados. As equipes deveriam desenvolver apenas um protótipo funcional. Diante dessa premissa, as equipes tinham que gerar um relatório fictício representando uma avaliação, que poderia ser no formato $\mathrm{html}$, pdf ou doc.

(2) Requisitos não funcionais. Foram definidos cinco RNF relacionados ao design e à estética do protótipo: (i) design responsivo, refere-se à possibilidade do uso do software em diversos dispositivos, tais como notebooks, celulares e tablets; (ii) design amigável, refere-se à uma interface intuitiva e com aparência atrativa; (iii) familiaridade, refere-se a frases e conceitos familiares ao usuário; (iv) consistência e padronização, que se referem à forma de apresentação dos dados e leiaute das páginas do protótipo, que devem seguir um padrão; e (v) avisos, que se referem às mensagens de aviso das ações e das navegações feitas pelo usuário no protótipo. 
Ao final as equipes devem apresentar um protótipo funcional que contempla os requisitos elicitados. Dessa forma, a técnica de prototipagem é usada por todas as equipes, pois é por meio dela que as equipes apresentam o resultado da ER.

\begin{tabular}{|c|c|c|c|c|}
\hline \multicolumn{3}{|c|}{ O Requisito está disponível no protótipo? } & Sim & Não \\
\hline \multirow{5}{*}{ 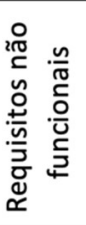 } & \multicolumn{2}{|c|}{ Linguagem do usuário (frases e conceitos familiares ao usuário } & & \\
\hline & \multicolumn{2}{|c|}{ Consistência e padronização } & & \\
\hline & \multicolumn{2}{|c|}{ Mensagens de aviso } & & \\
\hline & \multicolumn{2}{|c|}{ Estética e design atraentes } & & \\
\hline & \multicolumn{2}{|c|}{ Design da página responsido } & & \\
\hline \multirow{14}{*}{ 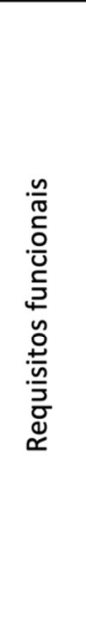 } & \multirow{2}{*}{ Desempenho } & Eficiência & & \\
\hline & & Eficácia & & \\
\hline & \multirow{4}{*}{ Comportamento } & Postura (frequência, pontualidade, linguagem) & & \\
\hline & & Comportamento sob pressão & & \\
\hline & & Comportamento ético & & \\
\hline & & Comportamento em relação aos desafios & & \\
\hline & \multirow{7}{*}{$\begin{array}{c}\text { Habilidades } \\
\text { sociais e pessoais }\end{array}$} & Resolução de problemas & & \\
\hline & & Capacidade de comunicação & & \\
\hline & & Proatividade & & \\
\hline & & Colaboração com a equipe & & \\
\hline & & \begin{tabular}{|l|} 
Relacionamento com a equipe \\
\end{tabular} & & \\
\hline & & Aderência aos valores e diretrizes da empresa & & \\
\hline & & Relacionamento interpessoal & & \\
\hline & & Gerar relatório da avaliação & & \\
\hline
\end{tabular}

Figura 3 - Requisitos elicitados

Fonte: elaborado pelo autor.

\subsubsection{Plano de Atividades e de Interação com as Equipes}

Foram definidas quatro interações para avaliação das atividades e dos resultados das equipes.

(1) Primeira. É apresentada a proposta geral do projeto. Nessa etapa as equipes são formadas e designadas à um tipo de equipe e é apresentado o cronograma de interações e atividades com duração de quatro meses.

(2) Segunda. Nesta interação todas as equipes devem usar a técnica de entrevistas para a ER. Os requisitos funcionais e não-funcionais são apresentados aos grupos, e os critérios para a elaboração do protótipo. Esperase com esta interação que as equipes tenham um entendimento dos requisitos do software para iniciar o desenvolvimento do protótipo. Foi solicitado também, que as equipes do tipo dois e três criassem histórias de usuário a partir dessa visão inicial do projeto, que deveriam ser entregues na terceira interação.

(3) Terceira. Nesta interação são usadas as técnicas entrevista, brainstorming e histórias de usuário. A proposta é verificar o trabalho desenvolvido pelas equipes desde a última interação, analisar a especificação dos requisitos elaborada pelas equipes e esclarecer dúvidas. Para isso, se estabeleceu as seguintes etapas: validações das histórias de usuários, entrevista com o cliente e realização de brainstorming, este último para atualizar as histórias de usuário. Durante essas etapas o pesquisador deve avaliar o envolvimento da equipe na execução das atividades, a comunicação e o comprometimento da equipe.

(4) Quarta. Esta é a última interação e se estabeleceu as seguintes etapas: as equipes apresentam as histórias de usuários atualizadas, a partir do resultado do brainstorming; as equipes entregam a versão final do protótipo; aqui também o pesquisador avalia o envolvimento da equipe na execução das atividades, a comunicação e o comprometimento da equipe. 


\section{Apresentação do Caso}

Nesta seção são descritas as características do caso. Primeiro são apresentadas as características das equipes, e em seguida as variáveis externas ao estudo e que se buscou manter constantes para não influenciar as análises.

\subsection{Características das Equipes}

A partir dos 53 alunos foram formadas 14 equipes, sendo 11 com quatro alunos e três com três alunos. Fez-se um levantamento do histórico de desempenho escolar de cada aluno com objetivo de caracterizar o desempenho escolar das equipes para análise conjunta com os resultados. Na primeira interação com as equipes foi constituída a formação das equipes aleatoriamente e realizada a designação do tipo de equipe. Os resultados estão apresentados na tabela 1

\begin{tabular}{|c|c|c|c|c|c|c|c|c|c|c|c|c|c|c|}
\hline \multirow[t]{2}{*}{ Características } & \multicolumn{14}{|c|}{ Equipes } \\
\hline & E1 & E2 & $\mathbf{E 3}$ & $\mathbf{E} 4$ & E5 & E6 & E7 & E8 & E9 & E10 & E11 & E12 & E13 & E14 \\
\hline No. alunos & 4 & 4 & 4 & 3 & 4 & 4 & 4 & 4 & 4 & 4 & 4 & 4 & 3 & 3 \\
\hline $\begin{array}{l}\text { Desempenho } \\
\text { escolar }\end{array}$ & 5,2 & 7,5 & 8,4 & 9,0 & 6,1 & 5,3 & 6,1 & 7,4 & 7,7 & 7,4 & 4,0 & 7,4 & 5,8 & 7,1 \\
\hline Tipo de equipe & $\mathrm{C}$ & $\mathrm{C}$ & $\mathrm{E}$ & $\mathrm{E}$ & $\mathrm{E}$ & $\mathrm{C}$ & $\mathrm{E}$ & $\mathrm{C}$ & $\mathrm{E}$ & $\mathrm{C}$ & $\mathrm{E}$ & $\mathrm{C}$ & $\mathrm{C}$ & $\mathrm{E}$ \\
\hline
\end{tabular}

\subsection{Variáveis Externas ao Estudo}

Para a execução do estudo procurou-se manter constante as variáveis que não fazem parte do modelo de referência desta pesquisa, de modo a permitir que apenas a participação da equipe influenciasse o processo de elicitação de RF e RNF. Entre as variáveis que foram mantidas constantes destacam-se: (1) a condução das atividades foi realizada pelo pesquisador em todas as equipes; (2) a forma de avaliação das equipes também foi realizada pelo pesquisador; (3) os requisitos a serem elicitados foram apresentados às equipes de forma similar; (4) o prazo das atividades foi igual para todas as equipes, ou seja, um semestre letivo para o desenvolvimento do projeto com quatro interações obrigatórias com o pesquisador, cada interação com igual tempo de duração entre as equipes; (5) os recursos tecnológicos utilizados foram os mesmos, ou seja, utilizou-se GitHub Pages (https://pages.github.com) para desenvolvimento do projeto, que é uma plataforma gratuita na qual vários usuários podem trabalhar no mesmo projeto compartilhando as informações; e (6) o número de alunos por equipe foi praticamente o mesmo, pois foram formadas equipes com quatro, sendo três equipes com três alunos devido ao número ímpar de alunos na turma.

\section{Apresentação dos Resultados}

Na tabela 2 são apresentados os resultados da ER obtidos pelas equipes com os protótipos, em relação às proposições de pesquisa P1 e P2. Nessas proposições foi analisada a influência da participação dos membros da equipe na qualidade da elicitação dos RF e RNF. Ambos os grupos - controle e experimental - utilizaram técnicas combinadas na ER. No grupo experimental todos os alunos (membros da equipe) participaram das interações com o cliente e no grupo controle apenas o PO teve a interação com o cliente. 


\begin{tabular}{|c|c|c|c|c|}
\hline Grupo & $\begin{array}{c}\text { Partici- } \\
\text { pação }\end{array}$ & Equipe & $\begin{array}{c}\text { Proposição P1 } \\
\text { RF }\end{array}$ & $\begin{array}{c}\text { Proposição P2 } \\
\text { RNF }\end{array}$ \\
\hline Controle & Só o PO & E01 & 0 & 1 \\
\hline Controle & Só o PO & E02 & 2 & 1 \\
\hline Controle & Só o PO & E06 & 1 & 1 \\
\hline Controle & Só o PO & E08 & 1 & 1 \\
\hline Controle & Só o PO & E10 & 1 & 0 \\
\hline Controle & Só o PO & E12 & 1 & 0 \\
\hline \multirow[t]{2}{*}{ Controle } & Só o PO & E13 & 1 & 1 \\
\hline & \multicolumn{2}{|c|}{ Desempenho grupo controle } & $50,0 \%$ & $35,7 \%$ \\
\hline Experimental & Todos & E03 & 2 & 0 \\
\hline Experimental & Todos & E04 & 2 & 2 \\
\hline Experimental & Todos & E05 & 1 & 1 \\
\hline Experimental & Todos & E07 & 1 & 0 \\
\hline Experimental & Todos & E09 & 1 & 1 \\
\hline Experimental & Todos & E11 & 1 & 1 \\
\hline \multirow[t]{2}{*}{ Experimental } & Todos & E14 & 2 & 2 \\
\hline & \multicolumn{2}{|c|}{ Desempenho grupo experimental } & $71,4 \%$ & $50,0 \%$ \\
\hline Diferença & & & $21,4 \%$ & $14,3 \%$ \\
\hline
\end{tabular}

Tabela 2 - Proposições P1 e P2.

Fonte: dados da pesquisa.

O desempenho dos grupos foi calculado pela somatória dos níveis de qualidade obtidos pelas equipes, divido pela somatória máxima possível. Exemplo: desempenho dos grupos de controle em relação aos RF foi de $50 \%$, ou seja, $7(0+2+1+1+1+1+1)$ dividido por $14(2+2+2+2+2+2+2)$.

O resultado esperado, em ambas as proposições, era que no grupo experimental a elicitação dos RF e RNF fossem de qualidade superior ao do grupo controle. Os resultados confirmaram as proposições. Na proposição um (P1), em que era esperado uma qualidade superior nos RF, as equipes do grupo experimental tiveram desempenho $21,4 \%$ superior ao grupo controle. De forma semelhante, na proposição dois (P2), em que era esperado uma qualidade superior nos RNF, as equipes do grupo experimental tiveram desempenho $14,3 \%$ superior ao grupo controle.

\section{Análise e Discussão dos Resultados}

A análise dos resultados foi feita por meio da variável de controle da pesquisa, constituída por dois indicadores: motivação e comunicação. A análise desses dois indicadores foi feita a partir de dados coletados nas interações com as equipes e nos questionários anônimos submetidos aos alunos.

$\mathrm{Na}$ tabela 3 são apresentadas as equipes analisadas nas proposições P1 e P2 e as variáveis de controle utilizadas para análise. 


\begin{tabular}{ccccccc}
\hline Grupo & Equipe & $\begin{array}{c}\text { P1 } \\
\text { (RF) }\end{array}$ & $\begin{array}{c}\text { P2 } \\
\text { (RNF) }\end{array}$ & Motivação & Comunicação & $\begin{array}{c}\text { Desempenho } \\
\text { escolar }\end{array}$ \\
\hline Controle & E01 & 0 & 1 & 1 & 1 & 5,2 \\
Controle & E02 & 2 & 1 & 1 & 1 & 7,5 \\
Controle & E06 & 1 & 1 & 2 & 1 & 5,3 \\
Controle & E08 & 1 & 1 & 1 & 1 & 7,4 \\
Controle & E10 & 1 & 0 & 1 & 0 & 7,4 \\
Controle & E12 & 1 & 0 & 1 & 1 & 7,4 \\
Controle & E13 & 1 & 1 & 2 & 2 & 5,8 \\
\cline { 2 - 7 } Desempenho grupo controle & $50 \%$ & $35,7 \%$ & $64,3 \%$ & $50,0 \%$ & 6,6 \\
Experimental & E03 & 2 & 0 & 2 & 2 & 8,4 \\
Experimental & E04 & 2 & 2 & 2 & 2 & 9,0 \\
Experimental & E05 & 1 & 1 & 2 & 2 & 6,1 \\
Experimental & E07 & 1 & 0 & 1 & 0 & 6,1 \\
Experimental & E09 & 1 & 1 & 2 & 1 & 7,7 \\
\cline { 2 - 7 } Experimental & E11 & 1 & 1 & 2 & 1 & 4,0 \\
Experimental & E14 & 2 & 2 & 1 & 2 & 7,1 \\
Desempenho grupo experimental & $71,4 \%$ & $50 \%$ & $85,7 \%$ & $71,4 \%$ & 6,9 \\
\hline Diferença & & $21,4 \%$ & $14,3 \%$ & $21,4 \%$ & $21,4 \%$ & 0,3 \\
\hline
\end{tabular}

Tabela 3 - Variáveis de controle.

Fonte: dados da pesquisa.

Motivação. A motivação, avaliada pela assiduidade e pela frequência dos alunos nas interações, foi superior no grupo experimental em $21,4 \%$. É plausível atribuir essa motivação à participação de todos da equipe no processo de ER. Esse resultado vai ao encontro do que é destacado na Teoria Z (OUCHI, 1982), que enfatiza que o sucesso do processo administrativo está ligado à motivação humana e não simplesmente à tecnologia. Essa teoria baseiase no estilo de gerenciamento participativo, na qual os colaboradores são motivados por um senso de comprometimento, oportunidade e progresso.

Comunicação. A comunicação, avaliada em dois aspectos - entre os membros da equipe e entre a equipe e o cliente - também foi superior no grupo experimental em $21,4 \%$. É plausível atribuir esse resultado a menor incidência de ruído na comunicação. Esse resultado vai ao encontro do modelo básico de comunicação em projetos destacado pelo PMBoK (PMI, 2017), que exemplifica fatores denominados de ruídos no processo de transmissão da mensagem.

Desempenho escolar. A diferença no desempenho escolar dos grupos de controle e experimental foi muito baixa, ou seja, apenas 0,3 . As equipes do grupo experimental tiveram apenas uma média $4,5 \%$ superior ao do grupo de controle. O desempenho escolar da equipe neste estudo de caso não foi fator associado a uma melhor ER.

\section{Considerações Finais}

O objetivo desta pesquisa foi analisar a participação da equipe na qualidade de elicitação de requisitos funcionais (RF) e requisitos não funcionais (RNF). Para atingir estes objetivos, efetuou-se uma pesquisa qualitativa e exploratória com base em um estudo de caso envolvendo 53 alunos de graduação durante o segundo semestre de 2019. Cabe destacar que a pesquisa apresenta limitações das quais se destacam: (1) viés do pesquisador, pois as análises dos dados coletados foram interpretados pelo pesquisador, atribuindo subjetividade ao processo; (2) 
ambiente educacional, pois apesar dos cuidados em relação às variáveis da pesquisa e a escolha adequada de alunos de um curso de Sistemas de informação, o ambiente educacional apresenta diferenças do ambiente empresarial, que possui projetos de desenvolvimento de software sujeitos a um contexto diferenciado em termos de objetivos, estratégias, conflitos e demais variáveis do ambiente empresarial; e (3) o método utilizado que permite um entendimento maior do caso analisado, porém não permite a generalização dos resultados.

Os resultados permitiram confirmar, especificamente para o caso analisado, que a maior participação dos membros da equipe no processo de ER melhorou a completude e a consistência dos RF e RNF elicitados. Além disso, foi possível observar variáveis de controle que se associaram positivamente a esse resultado: motivação e comunicação. Sugerem-se que essas variáveis sejam analisadas em futuras pesquisas, tanto exploratórias como de caráter confirmatória. O desempenho escolar não se associou aos resultados da pesquisa, porém sugere-se que em futuras pesquisas essa variável seja mantida constante ao longo do estudo, ou seja, que as equipes sejam formadas de modo que todas apresentem um desempenho escolar similar.

\section{Referências}

ALVERTIS, I.; PAPASPYROS, D.; KOUSSOURIS, S.; MOUZAKITIS, S.; ASKOUNIS, D. Using crowdsourced and anonymized personas in the requirements elicitation and software development phases of software engineering. 11th International Conference on Availability, Reliability and Security (ARES), pp. 851856, 2016. IEEE. doi: 10.1109/ARES.2016.71.

ANGELIS, G.; FERRARI, A.; GNESI, S.; POLINI, A. Requirements elicitation and refinement in collaborative research projects. Journal of Software: Evolution and Process, v. 30, n. 12, p. 1-18, 2018. doi: $10.1002 / \mathrm{smr} .1990$.

ARAUJO, R.; ANJOS, E.; SILVA, D. Trends in the use of design thinking for embedded systems. 15th International Conference on Computational Science and Its Applications, pp. 82-86, 2015. doi: 10.1109/ICCSA.2015.25.

ASGHAR, A.; TABASSUM, A.; BHATTI, S.; JADI, A. Impact and challenges of requirements elicitation prioritization in quality to agile process: Scrum as a case scenario. 2017 International Conference on Communication Technologies (ComTech), pp. 50-55, 2017. IEEE. 10.1109/COMTECH.2017.8065749.

BABAR, A.; BUNKER, D.; GILL, A. Investigating the relationship between business analyst's competency and is requirements elicitation: A thematic analysis approach. Communications of The Association for Information Systems, v. 42, n. 1, p. 334-362, 2018. Association for Information Systems. doi: 10.17705/1CAIS.04212.

BATOOL, A.; MOTLA, Y.; HAMID, B.; ASGHAR, S.; RIAZ, M.; MUKHTAR, M.; AHMED, M. Comparative study of traditional requirement engineering and agile requirement engineering. International Conference on Advanced Communication Technology, ICACT, pp. 1006-1014, 2013.

BIGGS, J. B.; TANG, C. S. Effective teaching and learning for today's universities. In: Biggs, J. B.; Tang. Teaching for quality learning at university. New York, USA: McGraw-Hill Education, p. 1-16, 2011.

BOEHM, B. Requirements that handle ikiwisi, cots, and rapid change. Computer, v. 33, n. 7, p. 99-102, 2000.

BOURQUE, P.; FAIRLEY, R. E. (2014). Guide to the Software Engineering Body of Knowledge (SWEBOK): Version 3.0. Los Alamitos, CA, USA: IEEE Computer Society Press, 2014.

BUITRON, S. L.; FLORES-RIOS, B. L.; PINO, F. J. Elicitación de requisitos no funcionales basada en la gestión de conocimiento de los stakeholders. Revista Chilena de Ingeniería, v. 26, n. 1, p. 142-156, 2018. doi: 10.4067/S0718-33052018000100142.

CERVO, A. L.; BERVIAN, P. A.; SILVA, R. Metodologia científica (6a ed.). São Paulo: Pearson Prentice Hall, 2007.

CRESWELL, J. Projeto de pesquisa: métodos qualitativo, quantitativo e misto. Artmed, 2007. 
CURCIO, K. D. C.; NAVARRO, T.; MALUCELLI, A.; REINEHR, S. Requirements engineering: a systematic mapping study in agile software development. Journal of Systems and Software, v. 139, n. 1, p. 32-50, 2018. doi: 10.1016/j.jss.2018.01.036.

DARDENNE, A.; VAN LAMSWEERDE, A.; FICKAS, S. Goal-directed requirements acquisition. Science of Computer Programming, v. 20, n. 1-2, p. 3-50, 1993.

EISENHARDT, K. M. Building theories from case study research. Academy of Management Review, v.14, n. 4, p. 532-550, 1989.

EL-NAJAR, T.; AHMAD, I.; ALKANDARI, M.; EASYCOMM. A framework and tool to solve client communication problem in agile development. International Journal of Computer Science, v. 46, n. 1, p. 1-12, 2019.

FERNANDES, J.; DUARTE, D.; RIBEIRO, C.; FARINHA, C.; PEREIRA, J.; Da SILVA, M. Ithink: a gamebased approach towards improving collaboration and participation in requirement elicitation. Procedia Computer Science, v. 15, n. 1, p. 66-77, 2012. doi: 10.1016/j.procs.2012.10.059.

FRANKEN, S.; KOLVENBACH, S.; PRINZ, W.; ALVERTIS, I.; KOUSSOURIS, S. Cloud teams: Bridging the gap between developers and customers during software development processes. Procedia Computer Science, v. 68, p. 188-195, 2015. doi: 10.1016/j.procs.2015.09.234.

GILL, K.; ZAIDI, A.; KIANI, M. Eliciting futuristic end-user requirements through contributory appreciative inquiry. IEEE-National Software Engineering Conference, Rawalpindi, pp. 49-54, 2014. doi: 10.1109/NSEC.2014.6998240.

GONZALES, C. K.; LEROY, G. Eliciting user requirements using appreciative inquiry. Empirical Software Engineering, v. 16, n. 6, p. 733-772, 2011. doi: 10.1007/s10664-011-9156-X.

GROTTA, A. Aprendizagem baseada em projeto ágil para educação em programação de computadores no ensino superior brasileiro. Dissertação - Escola de Artes, Ciências e Humanidades, Universidade de São Paulo, São Paulo, 2018.

HICKEY, A.; DAVIS, A. Elicitation technique selection: How do experts do it? Proceedings of the 11th IEEE International Conference on Requirements Engineering, pp. 169-178, 2003. IEEE. doi: 10.1109/ICRE.2003.1232748.

HIDALGA, A.; HARDISTY, A.; JONES, A. Scram-ck: applying a collaborative requirement engineering process for designing a web-based e-science toolkit. Requirements Engineering, v. 21, n. 1, p. 107-129, 2016. doi: 10.1007/s00766-014-0212-0.

HOFMANN, H. F.; LEHNER, F. Requirements engineering as a success factor in software projects. IEEE Computer Society Press, v. 18, n. 4, p. 58-66, 2001. doi: 10.1109/MS.2001.936219.

JIA, J.; CAPRETZ, L. Direct and mediating influences of user-developer perception gaps in requirements understanding on user participation. Requirements Engineering, v. 4, p. 14-04, 2017.

KNAUSS, E.; YUSSUF, A.; BLINCOE, K.; DAMIAN, D.; KNAUSS, A. Continuous clarification and emergent requirements flows in open-commercial software ecosystems. Requirements Engineering, v. 23, n. 1, p. 97-117, 2018. Springer London. doi: 10.1007/s00766-016-0259-1.

LAMSWEERDE, A. Requirements engineering in the year 00: a research perspective. Proceedings of the $\mathbf{2 2}^{\text {nd }}$ International Conference on Software Engineering - Icse '00, pp. 5-19, 2000. doi: $10.1145 / 337180.337184$.

LIM, S.; FINKELSTEIN, A. Stakerare: Using social networks and collaborative filtering for large-scale requirements elicitation. IEEE Transactions on Software Engineering, v. 38, n. 3, p. 707-735, 2012. doi: 10.1109/TSE.2011.36.

LIU, J. Y. C.; CHEN, H.G. CHEN, C. C.; SHEU, T. S. Relationships among interpersonal conflict, requirements uncertainty, and software project performance. International Journal of Project Management, v. 29, n. 5, p. 547-556, 2011. doi. 10.1016/j.ijproman.2010.04.007

MARIA, H.; ALI, Z. Requirement elicitation techniques for open source systems: A review. International Journal of Advanced Computer Science and Applications (IJACSA), v. 9, n. 1, p. 330-334, 2018. doi: $\underline{10.14569 / \text { IJACSA.2018.090145 }}$ 
MARSHALL, A.; GAMBLE, R. Gauging influence in software development teams. IEEE Frontiers in Education Conference, pp. 1-8, 2015. doi. 10.1109/FIE.2015.7344106

MELO, C.; CRUZES, D. S.; KON, F.; CONRADI, R. Agile team perceptions of productivity factors. 2011 Agile Conference, pp. 57-66, 2011. doi: 10.1109/AGILE.2011.35.

MISHRA, D.; MISHRA, A.; YAZICI, A. Successful requirement elicitation by combining requirement engineering techniques. In First International Conference on the Applications of Digital Information and Web Technologies (ICADIWT), pp. 258-263, 2008. IEEE. doi: 10.1109/ICADIWT.2008.4664355

NUSEIBEH, B.; EASTERBROOK, S. Requirements engineering: A roadmap. Proceedings of the Conference on The Future of Software Engineering, pp. 35-46, 2000. doi: 10.1145/336512.336523.

OUCHI, W. G. Teoria Z: como as empresas podem enfrentar o desafio japonês. São Paulo, Fundo Educativo Brasileiro, 1982.

PACHECO, C.; GARCIA, I. A systematic literature review of stakeholder identification methods in requirements elicitation. Journal of Systems and Software, v. 85, n. 9, p. 2171-2181, 2012. Elsevier BV. doi: 10.1016/j.jss.2012.04.075.

PANDEY, D.; SUMAN, U.; RAMANI, A. An effective requirement engineering process model for software development and requirements management. International Conference on Advances in Recent Technologies in Communication and Computing, pp. 287-291, 2010. doi: 10.1109/ARTCom.2010.24.

PMI. A Guide to the Project Management Body of Knowledge - PMBOK Guide, $6^{\text {th }}$ ed. Pennsylvania: Project Management Institute, 2017.

PRESSMAN, Roger S.; MAXIM, Bruce R.. Engenharia de software: uma abordagem profissional. 8 ed. Porto Alegre: AMGH, 2016.

ROMERO, M.; VIZCAÍNO, A.; PIATTINI, M. Teaching requirements elicitation within the context of global software development. Mexican International Conference on Computer Science, 232-239, 2009. doi: 10.1109/ENC.2009.29.

SADIQ, M.; GHAFIR, S.; SHAHID, M. An approach for eliciting software requirements and its prioritization using analytic hierarchy process. International Conference on Advances in Recent Technologies in Communication and Computing, 790-795, 2009. doi: 10.1109/ARTCom.2009.58.

SOMMERVILLE, I. Software engineering, 10 $10^{\text {th }}$ edition. Pearson, 2016.

VÄÄTÄJÄ, H.; HEIMONEN, T.; TIITINEN, K.; HAKULINEN, J.; TURUNEN, M.; NIEMINEN, H.; PAUNONEN, H.; RUOTSALAINEN, J.; OKSANEN, J.; LINDBORG, I. Opportunities and needs for logged usage data analytics of complex industrial systems. European Conference in Information Systems, 2016.

VIJAYAN, J.; RAJU, G.; JOSEPH, M. Collaborative requirements elicitation using elicitation tool for small projects. International Conference on Signal Processing, Communication, Power and Embedded System (SCOPES), 340-344, 2016. doi: 10.1145/10.1109/SCOPES.2016.7955848.

YOUNAS, M.; JAWAWI, D.; GHANI, I.; AND KAZMI, R. Non-functional requirements elicitation guideline for agile methods. Journal of Telecommunication, Electronic and Computer Engineering, v. 9, n. 1, 137$142,2017$. 


\section{Apêndice A}

Neste apêndice é apresentado o instrumento utilizado na coleta de dados da variável de controle: comunicação. No decorrer das interações, a pesquisadora fez entrevistas semi estruturadas com os alunos para avaliação do andamento do projeto e comunicação da equipe. A partir das observações da pesquisadora e dos resultados dos questionários apresentados abaixo, foram avaliados os níveis de comunicação.

\section{Questionário para avaliação da comunicação: equipe do tipo controle:}

1. Como foi a comunicação com o (a) PO? Ele(a) conseguiu repassar as informações com segurança para a equipe?

2. Como foi a comunicação entre os alunos da equipe? Surgiram crises ou desentendimentos?

3. Quais foram os canais utilizados para a comunicação entre a equipe

\section{Questionário para comunicação: equipe do tipo experimental.}

1. Na equipe que você participou a interação com a cliente foi com toda a equipe. Qual a sua percepção da diferença entre grupos com a participação total da equipe ou apenas com o PO? Na sua equipe, houve um representante ou PO?

2. Como foi a comunicação entre os alunos da equipe? Surgiram crises ou desentendimentos?

3. Quais foram os canais utilizados para a comunicação entre a equipe?

Os demais questionamentos realizados para avaliação e controle do projeto foram os mesmos para ambos os tipos de equipes: controle e experimento:

\section{Questionário para avaliação da Motivação}

1. Todos os alunos da equipe participaram da elicitação de requisitos? Caso alguém não tenha participado, descreva o motivo. (Por favor, não identifique o nome da pessoa).

2. Algum aluno da sua equipe não participou do desenvolvimento do projeto? Descreva o motivo.

\section{Questionário para verificação dos apontamentos da reunião}

1. Como foi seu entendimento da proposta do projeto para o desenvolvimento do protótipo do software?

2. As técnicas utilizadas pela sua equipe foram suficientes para o desenvolvimento? $\mathrm{O}$ que pode ser melhorado? Sugira outras técnicas para elicitação de requisitos.

3. Quantas reuniões presenciais foram realizadas? Caso não fizeram reuniões presenciais, descreva o motivo. 